\title{
Degenerate Fermi and non-Fermi liquids near a quantum critical phase transition
}

\author{
S. Kambe ${ }^{1 \star}$, H. Sakai ${ }^{1}$, Y. Tokunaga ${ }^{1}$, G. Lapertot ${ }^{2}$, T. D. Matsuda ${ }^{1 \dagger}$, G. Knebel ${ }^{2}$, J. Flouquet ${ }^{2}$ \\ and R. E. Walstedt ${ }^{3}$
}

Recently there is renewed interest in quantum critical phase transitions (QCPT) at $T=0 \mathrm{~K}$ in metallic strongly correlated electron systems. From early experimental results ${ }^{1-4}$, the QCPT in the Kondo-lattice compound $\mathrm{YbRh}_{2} \mathrm{Si}_{2}$ is not a case of the ordinary spin density wave (SDW) instability observed in Ce-based Kondo lattices ${ }^{5}$, but a candidate for a novel locally critical case ${ }^{6-8}$. Here, we observe that coexisting, static Fermi liquid (FL) and non-Fermi liquid (NFL) states are a key feature of the QCPT in $\mathrm{YbRh}_{2} \mathrm{Si}_{2}$. By means of nuclear magnetic resonance (NMR) spin-lattice relaxation time $\left(T_{1}\right)$ measurements on a single-crystalline sample, we find that the FL and NFL states are invariant, whereas their ratio in a crossover is field dependent near the QCPT. Such a pair of states has remained hidden in Ce compounds, owing presumably to the short lifetimes of the two states. We derive a scaling law for the occupation ratio of the two states, which could be widely applicable to Kondo-lattice systems.

The low-temperature heavy-fermion state of a Kondo lattice often exhibits a QCPT. In the heavy-fermion $\mathrm{YbRh}_{2} \mathrm{Si}_{2}$, the weak antiferromagnetic transition below $T_{N} \sim 70 \mathrm{mK}$ is easily driven to $T=0$ with a small applied magnetic field $H$ (ref. 1). At the critical magnetic field $H_{c}$ for $T_{N} \sim 0 \mathrm{~K}$, the $T$-dependence of physical properties shows NFL behaviour due to quantum critical fluctuations on approaching the QCPT (ref. 9). As a consequence of its tetragonal crystal structure (Fig. 1a), the electronic state of $\mathrm{YbRh}_{2} \mathrm{Si}_{2}$ is anisotropic ${ }^{10}$. Thus, the critical field $H_{\mathrm{c}} \sim 0.06 \mathrm{~T}$ and $\sim 0.66 \mathrm{~T}$ for $H$ perpendicular to the $c$ axis $(H \perp c)$ and parallel to the $c$ axis $(H \| c)$, respectively, indicating that the effective energy scale for quantum criticality is $\sim 10$ times larger for $H \| c$. Here we have chosen to apply $H$ along the $c$ axis, as the NFL behaviour can then be seen over a wide range of temperatures and fields.

In the present study, the $T$ - and $H \| c$-dependence of the ${ }^{29} \mathrm{Si}$ nuclear spin-lattice relaxation time $T_{1}$ has been measured for $45 \mathrm{mK} \leq T \leq 300 \mathrm{~K}$ and $0.45 \mathrm{~T} \leq H \leq 7.2 \mathrm{~T}$. The low natural abundance $(\sim 4.7 \%)$ of ${ }^{29} \mathrm{Si}\left(I=1 / 2, \gamma_{n} /(2 \pi)=845.77 \mathrm{~Hz} \mathrm{Oe}^{-1}, \gamma_{n}\right.$ : gyromagnetic ratio) has so far prevented highly accurate ${ }^{29} \mathrm{Si}$ NMR measurements in $\mathrm{YbRh}_{2} \mathrm{Si}_{2}$. For the present study a single-crystal sample has been prepared with the ${ }^{29} \mathrm{Si}$ isotope enriched to $52 \%$, improving the NMR sensitivity by a factor $\sim 11$.

$T_{1}$ for $H \| c$ is related to the dynamical magnetic susceptibility along the $a$ axis, $\operatorname{Im} \chi_{a}\left(q, \omega_{n}\right)$, according to the relation ${ }^{11}$

$$
1 / T_{1} T=2 \gamma_{n}^{2} \sum_{q} A_{a}^{2}(q) \operatorname{Im} \chi_{a}\left(q, \omega_{n}\right) / \omega_{n}
$$

where $A_{a}(q)$ is the transferred hyperfine coupling tensor along the $a$ axis at the Si site and $\omega_{n} /(2 \pi)=\gamma_{n} H /(2 \pi)$ is the NMR measurement frequency $(\sim \mathrm{MHz})$. The spin-lattice relaxation of the ${ }^{29} \mathrm{Si}$ is mainly driven by magnetic fluctuations at the $\mathrm{Yb}$ sites through transferred hyperfine coupling. Therefore, $1 / T_{1} T$ is a direct measure of $\operatorname{Im} \chi_{a}\left(q, \omega_{n}\right)$-that is, of the magnetic excitations at the $\mathrm{Yb}$ site. In a FL state, a Korringa-like relaxation $1 / T_{1} T \sim$ const. is expected, whereas in a NFL state, $1 / T_{1} T \sim T^{-\nu}$, leading to $\operatorname{Im} \chi_{a}\left(q, \omega_{n}\right) \rightarrow \infty$ as $T \rightarrow 0 \mathrm{~K}$, where the value of $v$ depends on the type of criticality ${ }^{12}$. For ${ }^{29} \mathrm{Si}(I=1 / 2)$ the time $t$-dependence of the spin-lattice relaxation curve for nuclear magnetization $M(t)$ in a homogeneous electronic state is a simple exponential with a unique $T_{1}$

$$
\frac{M(t)}{M(\infty)}=1-\exp \left(-t / T_{1}\right)
$$

where $M(\infty)$ corresponds to the equilibrium nuclear magnetization. If two electronic states, an S-state with short $T_{1 S}$ and an L-state with long $T_{1 \mathrm{~L}}$, coexist in the sample, then the relaxation curve is expressed by a two-component equation (Fig. 1b)

$$
\begin{gathered}
\frac{M(t)}{M(\infty)}=\frac{M_{\mathrm{L}}(\infty)}{M(\infty)}\left[R\left\{1-\exp \left(-t / T_{1 \mathrm{~S}}\right)\right\}+1-\exp \left(-t / T_{1 \mathrm{~L}}\right)\right] \\
M(\infty)=M_{\mathrm{S}}(\infty)+M_{\mathrm{L}}(\infty) \\
R=M_{\mathrm{S}}(\infty) / M_{\mathrm{L}}(\infty)
\end{gathered}
$$

where $M_{\mathrm{S}}(\infty)$ and $M_{\mathrm{L}}(\infty)$ are the S- and L-state equilibrium nuclear magnetizations, respectively. Equation (2) then corresponds to the cases $M_{\mathrm{S}}(\infty)=0$ or $M_{\mathrm{L}}(\infty)=0$. The ratio $R$ gives the proportion of S-state to L-state nuclear magnetization in the sample. Because $M_{\mathrm{S}, \mathrm{L}}(\infty)$ is proportional to the respective numbers of $\mathrm{Si}$ in each state, $R$ corresponds to the ratio of the NFL state population, $f_{\mathrm{NFL}}(T)$, to that of the FL state, $f_{\mathrm{FL}}(T)=1-f_{\mathrm{NFL}}(T)$; that is, $R=f_{\mathrm{NFL}}(T) / f_{\mathrm{FL}}(T)$, as is clarified below. In the present measurements, all relaxation data obtained are fitted well either by equation (2) or (3). Thus, there is no continuous distribution of $T_{1}$ in the present case; in particular, $M(t)$ results cannot be fitted with the stretched exponential function ${ }^{13}$.

Figure 2a shows semi-logarithmic plots of $1-M(t) / M(\infty)$ at the critical field $H=0.66 \mathrm{~T}$ for different temperatures. At high temperatures-for example, $1.05 \mathrm{~K}$-a clear straight line (equation (2)) is obtained, indicating a homogeneous state with

\footnotetext{
${ }^{1}$ Advanced Science Research Center, Japan Atomic Energy Agency, Tokai-mura, Ibaraki 319-1195, Japan, ${ }^{2}$ Service de Physique Statistique, Magnétisme et Supraconductivité, Institut Nanosciences et Cryogénie, CEA-Grenoble/Université J. Fourier Grenoble I, 17 rue des Martyrs, Grenoble 38054, France, ${ }^{3}$ Physics Department, The University of Michigan, Ann Arbor, Michigan 48109, USA. †Present address: Division of Physics, Tokyo Metropolitan University, Hachioji-shi, Tokyo 192-0397, Japan. *e-mail: kambe.shinsaku@jaea.go.jp
} 
a

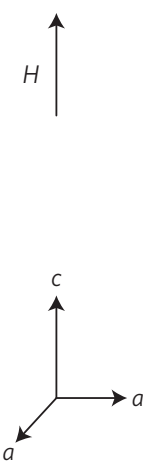

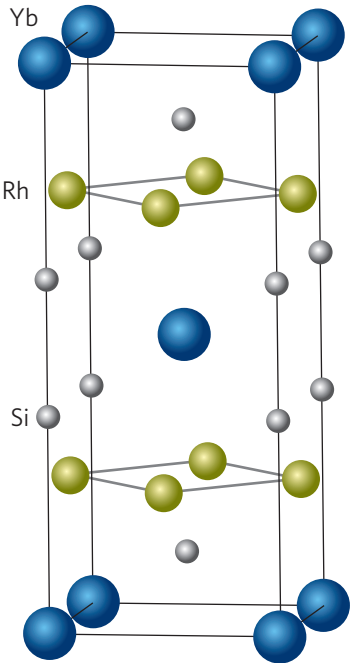

b

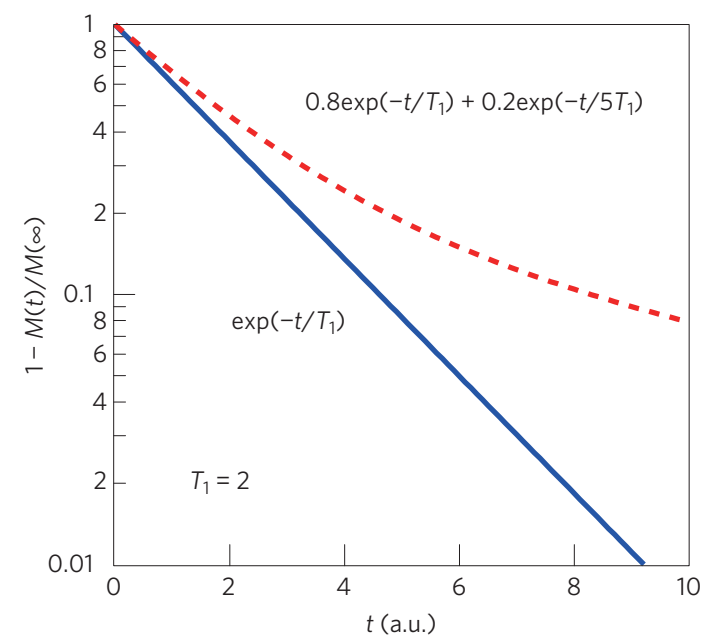

Figure 1| Tetragonal crystal structure of $\mathrm{YbRh}_{2} \mathrm{Si}_{2}$ and schematic nuclear relaxation $\left(T_{1}\right)$ curves for single and dual electronic states. a, Crystal structure (space group $14 / \mathrm{mmm}$ ) of $\mathrm{YbRh}_{2} \mathrm{Si}_{2}$. A magnetic field $\mathrm{H}$ is applied along the $\mathrm{c}$ axis. The local symmetry of the Si site is tetragonal ( $4 \mathrm{~mm}$ ) with four nearest-neighbour $\mathrm{Yb}$ atoms. By symmetry, in-plane antiferromagnetic fluctuations on the Yb sites cancel out at the Si site. $\mathbf{b}$, Relaxation curves for nuclear magnetization. The solid line represents the relaxation curve for a homogeneous state with a unique $T_{1}$ (equation (2)). The dashed line represents the relaxation curve for two coexisting states (equation (3) with $T_{1 \mathrm{~L}} / T_{1 \mathrm{~S}}=5$ and $R=4$ ). $T_{1}$ is taken as 2 in the units of time shown. As illustrated, a semi-logarithmic plot of $1-M(t) / M(\infty)$ shows a straight line for a homogeneous state, whereas, in contrast, a curved line is expected for two coexisting states.

a unique $T_{1}$. In contrast, as $T$ decreases, the $1-M(t) / M(\infty)$ curve is bent strongly in a fashion that is well fitted by the twocomponent equation (3), indicating that two different states appear simultaneously at lower temperatures.

Figure $2 \mathrm{~b}$ shows semi-logarithmic plots of $1-M(t) / M(\infty)$ at $T=45 \mathrm{mK}$ for different magnetic fields. A clear straight line at 7.2 $\mathrm{T}$ indicates a homogeneous electronic state at high magnetic fields. As the field decreases, plot curvature again indicates that two different states appear at low fields. It should be noted here that the NMR spectra of the FL and NFL states are indistinguishable (Supplementary Information).

Figure 3 shows the $T$-dependence of $1 / T_{1 \mathrm{~L}} T$ and $1 / T_{1 \mathrm{~S}} T$ at different magnetic fields. Because $1 / T_{1 \mathrm{~L}} T$ is roughly independent of $T$ at low temperatures, the L-state corresponds to the FL state. The weak $T$-dependence of $1 / T_{1 \mathrm{~L}} T$ may be a result of fine structure at the Fermi level ${ }^{14}$.

On the other hand for the S-state, $1 / T_{1 S} T \propto T^{-v} \quad(v \sim 1)$, which corresponds to the NFL state. In this compound, the static susceptibility along the $a$ axis, $\chi_{a}$, is also proportional to $\sim T^{-1}$ at low field ${ }^{9}$, thus $\chi_{a} \propto 1 / T_{1 S} T \propto T^{-1}$ for the NFL state, indicating that the NFL state has a localized nature ${ }^{15}$. Unique and $H$-independent $1 / T_{1} T$ above $3 \mathrm{~K}$ indicates simply that $\mathrm{YbRh}_{2} \mathrm{Si}_{2}$ is a nearly localized, uniform system at high temperatures.

The present results indicate that there is coexistence of FL and localized-NFL states in the sample around the QCPT. Remarkably, the $T$-dependences of $1 / T_{1 \mathrm{~L}} T$ and $1 / T_{1 \mathrm{~S}} T$ are independent of field within experimental error, indicating that the FL and NFL states are insensitive to applied magnetic field. In contrast, the ratio $R=f_{\mathrm{NFL}}(T) / f_{\mathrm{FL}}(T)$ between populations of the two states depends on both field and temperature. Figure $4 \mathrm{a}$,b illustrates the $H, T$ dependence of $R$. The NFL state is seen to be prominent at all temperatures around $H_{c}$, consistent with previous measurements ${ }^{1}$.

The $q$-dependence of the hyperfine coupling: $A_{a}(q)^{2}=A_{a}(0)^{2} \cos ^{2}\left(q_{x} / 2\right) \cos ^{2}\left(q_{y} / 2\right)$ at the Si site (Fig. 4c) mandates cancellation of commensurate antiferromagnetic excitations $\left(q_{x}= \pm \pi\right.$ and/or $q_{y}= \pm \pi$; ref. 16), and therefore $T_{1}$ is driven mainly by ferromagnetic excitations near $q_{x}=q_{y}=0$. Although the QCPT singularity (that is, NFL behaviour) occurs at an unidentified incommensurate antiferromagnetic wavevector ${ }^{17}$, ferromagnetic fluctuations mainly come into play here. The $H$-dependence of quantum-critical antiferromagnetic fluctuations generally shows a maximum around $H_{c}$; in contrast, ferromagnetic fluctuations are found to strengthen with decreasing $H \rightarrow 0$ in neutron scattering ${ }^{17}$ and NMR (ref. 18) measurements. Because both fluctuations contribute to $1 / T_{1} T$ through the $q$-dependent $A_{a}(q)$, the expected maximum of $1 / T_{1} T$ at $H_{c}$ (ref. 10) is blurred. The measured parameter $R$ characterizes the QCPT via integrated magnetic fluctuations which extend broadly over $q$-space owing to the localized magnetic character of $\mathrm{YbRh}_{2} \mathrm{Si}_{2}$. The dominant ferromagnetic fluctuations seem to be enhanced mainly in the FL state, which explains the large proportion of the FL state $\left(f_{\mathrm{FL}} \sim 0.4\right)$ observed at $H_{c}$.

Based on a Lorentzian approximation, $\operatorname{Im} \chi_{a}(q, \omega) / \omega=\pi \chi_{a} / \Gamma$ near $\omega \sim 0$ (ref. 19), equation (1) and the hyperfine coupling constant $A_{a}(0) \sim-0.20 \mathrm{kOe} / \mu_{\mathrm{B}}$ determined via Knight shift measurements (Supplementary Information), a characteristic magnetic fluctuation energy along the $a$ axis, $\Gamma \sim 3 \mathrm{~K}$, is obtained for the FL state. This rather small $\Gamma$ value compared with the bare Fermi energy $\sim 10^{4} \mathrm{~K}$ indicates that the Fermi liquid state is a highly renormalized heavy-fermion state. For the NFL state, the expression for the localized moment case ${ }^{15}, 1 / T_{1 \mathrm{~S}} \sim 1 / 3(2 \pi)^{0.5} A_{a}(0)^{2} /\left(\hbar J_{\mathrm{ex}}\right)$, yields a small exchange energy, $J_{\text {ex }} \sim 4 \mathrm{~K}$, reflecting quantum critical fluctuations with an energy comparable to $\Gamma$. The FL and NFL states are nearly degenerate near the QCPT, because $\Gamma$ and $J_{\text {ex }}$ have similar magnitudes.

The spatial size of each state cannot be estimated from NMR measurements, as it is a local probe. However, the state distribution must be stable for times $\Delta t \gg T_{1 \mathrm{~L}}$ in order for equation (3) to be valid. At the lowest temperatures, such a time period of stability is of the order of seconds. With excellent sample homogeneity, the FL and NFL states seem to mingle with each other very smoothly. The coexistence of localized and spatially extended fluctuations is indicated by both ESR (ref. 20) and Mössbauer ${ }^{21}$ measurements.

In a previous $T_{1}$ study for $H \perp c$ (ref. 18), the coexistence of two states was not reported. In the present study, two-state coexistence has also been confirmed for $H \| a$ (Supplementary Information). 

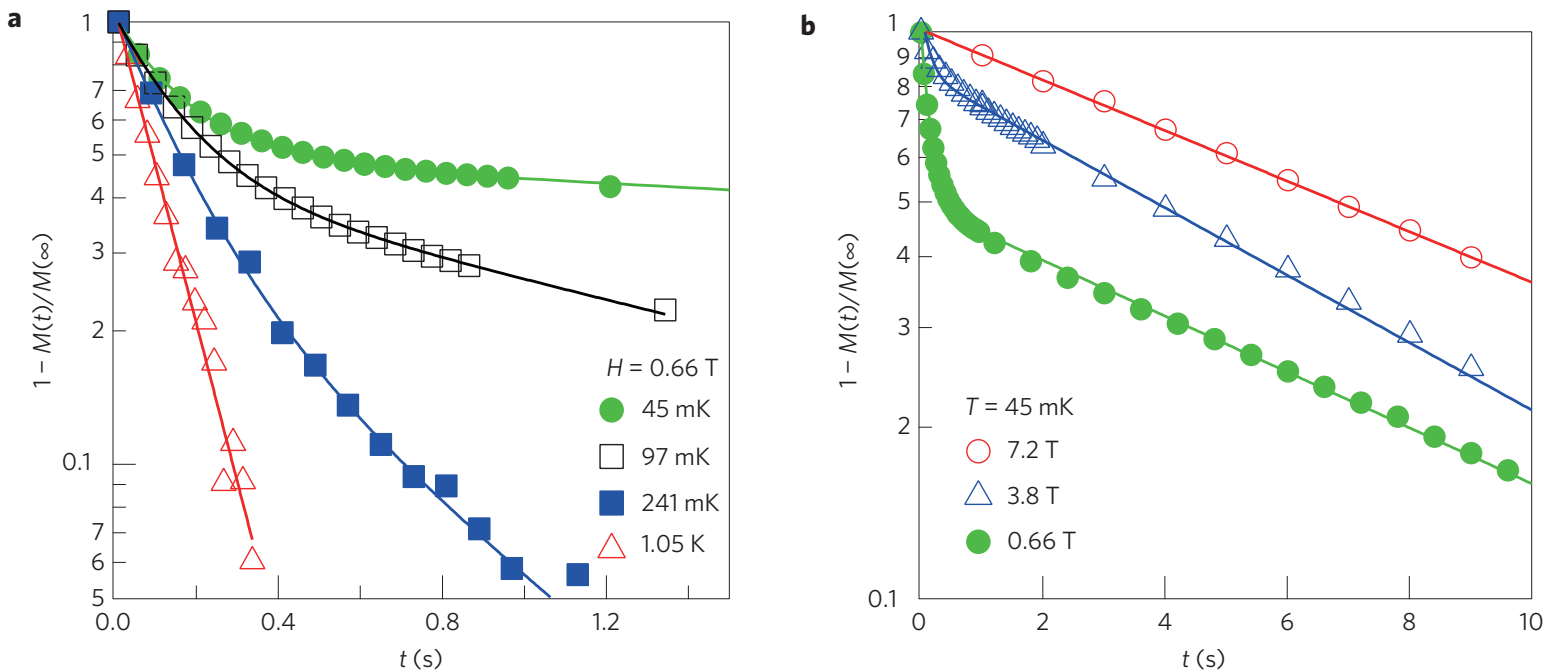

Figure 2 | Families of constant-field and constant-temperature nuclear relaxation curves exhibiting two-state (FL and NFL) behaviour near the QCPT. a, Nuclear magnetic relaxation curves at the critical field $\mathrm{H}=0.66 \mathrm{~T}$ at different temperatures. Solid lines are obtained by least squares fits to equation (2) for $1.05 \mathrm{~K}$ and to equation (3) for other temperatures. At $1.05 \mathrm{~K}$, the relaxation curve is a straight line, indicating a homogeneous (NFL) state. As temperature decreases, two-component relaxation curves appear, which are well fitted by equation (3), indicating the appearance of two different states. b, Nuclear relaxation curves at $T=45 \mathrm{mK}$ in different applied fields. Solid lines are obtained by least squares fits to equation (2) for $7.2 \mathrm{~T}$ and to equation (3) for 3.8 T and 0.66 T. At 7.2 T the relaxation curve is straight, indicating a homogeneous (FL) state. With decreasing field, nonlinear relaxation curves appear, which are well fitted by equation (3), indicating the presence of two different states.

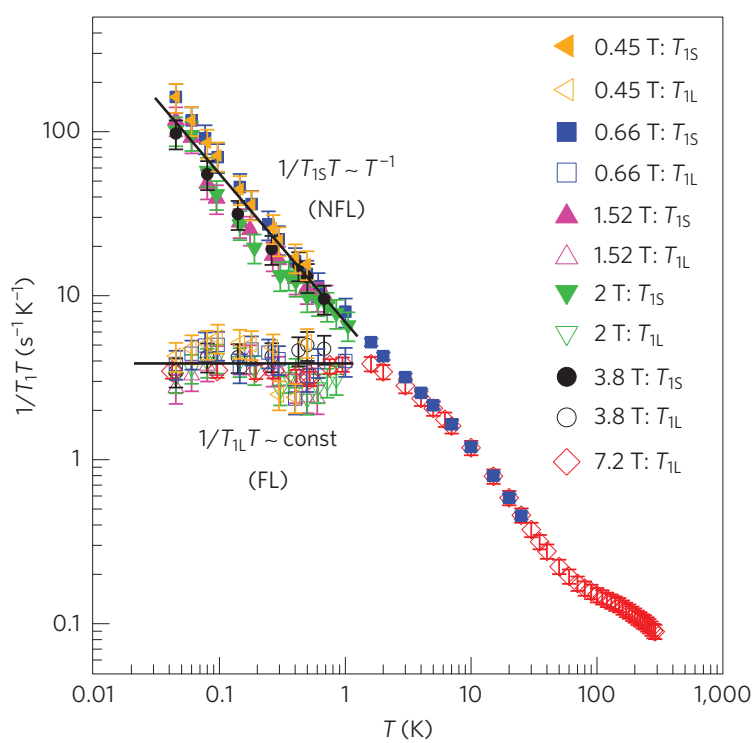

Figure 3 | Comprehensive plot of data for $1 / T_{1} T$ versus $T$ shows separate FL and NFL branches below $T \sim 1 \mathrm{~K}$. $T$-dependence of $1 / T_{1 S} T$ : (NFL state) and $1 / T_{1 L} T$ : (FL state) at different fields, as listed in the figure. Above $\sim 3 \mathrm{~K}$, there is a unique $T_{1}$, independent of field. Below $\sim 3 \mathrm{~K}, T_{1}$ shows a field dependence. With decreasing $T$ below $\sim 1 \mathrm{~K}$ at low fields, distinct values are resolved for $1 / T_{1 \mathrm{~S}} T$ and $1 / T_{1 \mathrm{~L}} T$. Remarkably, the values of $1 / T_{1 \mathrm{~S}} T$ and $1 / T_{1 \mathrm{~L}} T$ are independent of field at each temperature, with only the proportion $R$ between the two components changing with field. At the highest field 7.2 T, only the Fermi liquid state with $1 / T_{1 L} T=$ constant remains. Error bars are determined by least squares fits to the nuclear relaxation curves (Fig. 2) using equations ( 2 ) and ( 3 ).

However, such coexistence is restricted to a region near the QCPT (that is, at very low field and temperature) for $H \| a$, because $H_{c}$ and the energy scale are ten times smaller.

In proposed theoretical models (SDW (ref. 5), valence criticality ${ }^{22}$ and strong coupling ${ }^{23}$ ), only a single state has been considered and, thus, a unique $T_{1}$ is proposed. The local critical model ${ }^{6}$ implies coexisting local (NFL) and itinerant (FL) type excitations, which may be related to the present observations. However, static two-state equilibrium is not predicted in that model.

The two-fluid model ${ }^{24,25}$ for Ce-based heavy-fermion compounds assumes a state with Kondo liquid (FL) and spin liquid (NFL) components. This approach successfully modelled physical properties using the equation for the FL state population, $f_{\mathrm{FL}}(T)=f_{\mathrm{FL}}(0)\left(1-T / T^{*}\right)^{1.5}$, where $f_{\mathrm{FL}}(T)+f_{\mathrm{NFL}}(T)=1$. However, the two fluids concerned have never been observed separately, even at the SDW-QCPT (ref. 26), because Ce-case fluids in this state resonate with each other on a timescale $\tau$ which is very short.

Adopting the two-fluid approach, the parameter $R$ (equation (3)) may be written as $R_{2 \mathrm{fl}}(T)=f_{\mathrm{NFL}}(T) / f_{\mathrm{FL}}(T)=$ $f_{\mathrm{FL}}(0)^{-1}\left(1-T / T^{*}\right)^{-1.5}-1$, where $T^{*}$ is the coherence temperature ${ }^{25}$. $R_{2 f l}(T)$ is seen to give an approximate representation of the data curves in Fig. 4a, where behaviours in the limits $T \rightarrow 0$ and $T \rightarrow T^{*}$ are clearly dependent on the field $H$. We may account for this with a scaling relation for $R$ based on $R_{2 f 1}(T)$ of the form

$$
R_{\mathrm{SC}}=T^{\alpha} \Phi\left(H / T^{\beta}\right)
$$

where $\Phi$ is a scaling function, $\alpha=-1 \pm 0.2$ and $\beta=2.5 \pm 0.4$. Although $R_{2 \mathrm{fl}}(T)$ does not explicitly include scaling with field, equation (4) leads to approximate $H$-dependences for $T^{*} \simeq 0.64 H^{\gamma}(\gamma=1 / \beta \simeq 0.4 \pm 0.1)$ and for $f_{\mathrm{FL}}(0) \simeq 0.59 H^{0.19 \pm 0.05}$ (Supplementary Information). The scaling relation is confirmed experimentally in Fig. $4 \mathrm{~d}$ as $R T \simeq \Phi(x)$ versus $x=H / T^{2.5}$, with $\Phi(x)=0.73 x^{-0.4}\left\{2\left(1-1.55 x^{-0.4}\right)^{-1.5}-1\right\}$ calculated using $R_{2 \mathrm{fl}}(T)$ (see details in figure caption and Supplementary Information). It is most accurate at the low-temperature (large $x$ ) end.

Beyond the diminished singularity at $H_{c}$ noted above, the magnitude and $H$-dependence of $T^{*}$ are consistent with the reported characteristic temperature and $H$-dependence of effective mass $m^{*} \sim\left(H-H_{c}\right)^{-(0.33 \sim 0.5)} \sim 1 / T^{*}$ in $\mathrm{YbRh}_{2} \mathrm{Si}_{2}$ (ref. 1) and $\mathrm{YbRh}_{2}\left(\mathrm{Si}_{0.95} \mathrm{Ge}_{0.05}\right)_{2}$ (ref. 10). Such an $H$-dependence for $m^{*} \sim H^{-0.5}$ was also adopted for the Ce-based Kondo lattice-for example, $\mathrm{CeB}_{6}$ (ref. 27). 
a
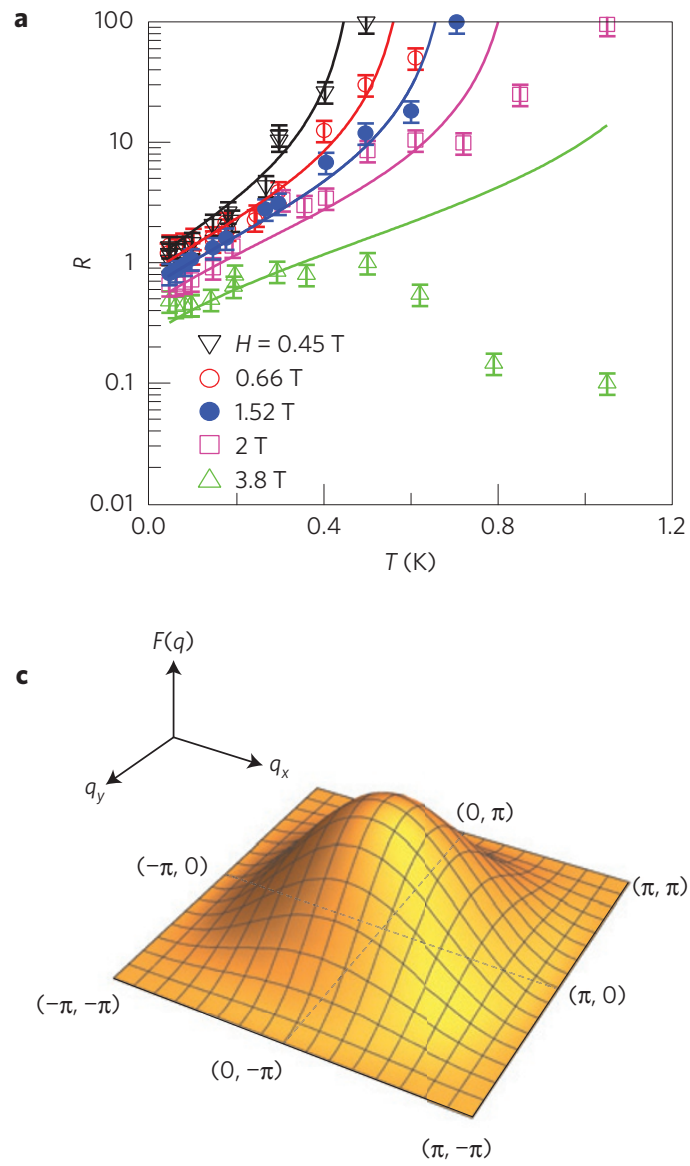

b

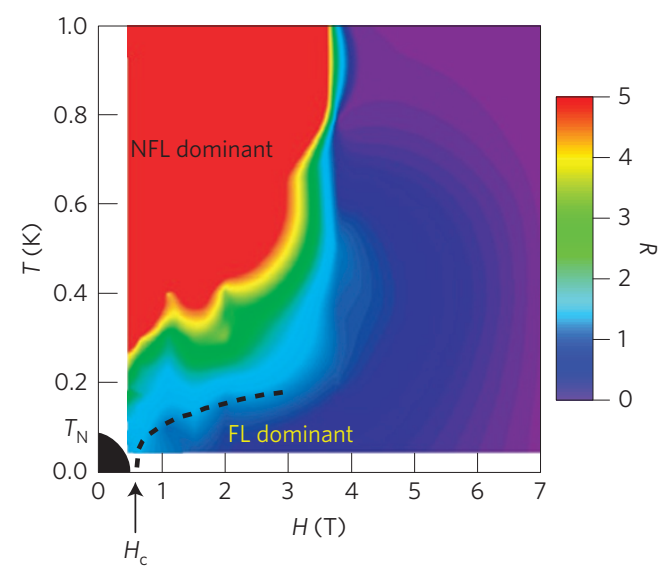

d

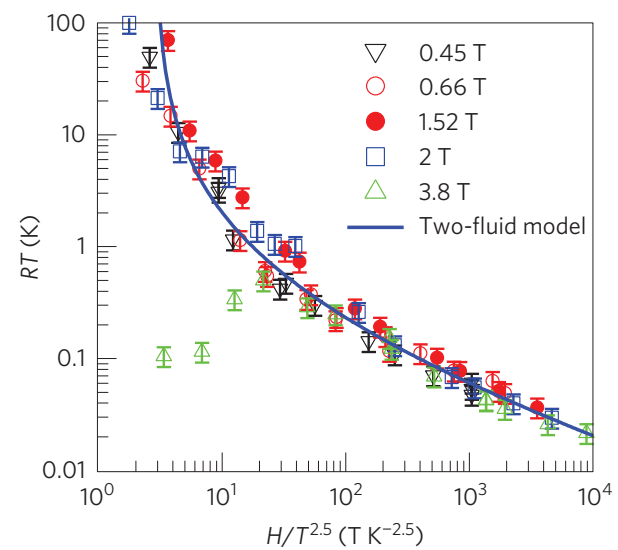

Figure 4 | Comprehensive plot, colour map and scaling relation for the ratio of NFL to FL populations $R$ versus $\boldsymbol{H}$ and $T$. $\mathbf{a}, T$-dependence of the proportion of NFL to FL state $R=M_{\mathrm{S}}(\infty) / M_{\mathrm{L}}(\infty)=f_{\mathrm{NFL}}(T) / f_{\mathrm{FL}}(T)$ at different fields. At $H=0.45$ and $0.66 \mathrm{~T}$, no trace of the FL state is observed; that is, $R \sim \infty$ above $0.6 \mathrm{~K}$. In contrast, at $H=7.2 \mathrm{~T}, R \sim 0$ below $\sim 1 \mathrm{~K}$ (thus, not shown). At 3.8 $\mathrm{T}$, the behaviour above $0.5 \mathrm{~K}$ is more complicated, because the system is on the boundary between the NFL and FL states (Supplementary Information). Solid lines are fitted curves using the two-fluid formula $R_{2 f 1}(T)$ (see text). Here, $T^{*} \simeq 0.64 H^{0.4}$ and $f_{\mathrm{FL}}(0) \simeq 0.59 H^{0.19}$ ( $T^{*}$ and $H$ are in units of kelvin and tesla, respectively). b, Colour map of the proportion $R$ of the NFL to FL state in the $(H, T)$ plane. In the red region, the NFL state is dominant, corresponding to the quantum critical regime. In the blue region the FL state is dominant. At low temperatures the FL state becomes dominant as field increases and, finally, above $\sim 4 \mathrm{~T}$, FL behaviour is observed for the whole temperature range. At any fixed temperature the proportion of FL to NFL states varies smoothly across the diagram, whereas $T_{1 \mathrm{~S}}$ and $T_{1 \mathrm{~L}}$ remain constant. The dashed line is the crossover between NFL and FL states previously determined in resistivity measurements ${ }^{1}$, which is qualitatively consistent with the present results. The crossover line corresponds to the boundary for $R \sim 1.5$. The black region indicates the antiferromagnetic ordering regime ${ }^{1}$. $\mathbf{c}, q$-dependence of hyperfine coupling at the Si site $F(q) \equiv\left(A_{a}(q) / A_{a}(0)\right)^{2}=\cos ^{2}\left(q_{x} / 2\right) \cos ^{2}\left(q_{y} / 2\right)$. As shown, $A_{a}(q)^{2}$ has a maximum at $\left(q_{x}, q_{y}\right)=(0,0)$; thus, the ferromagnetic contribution is predominant as compared with antiferromagnetic coupling in the $1 / T_{1} T$ measurements. $\mathbf{d}$, Scaling plot for the results from $\mathbf{a}$. $R$ obeys the scaling relation $R T=\Phi\left(H / T^{2.5}\right)$, with scaling function $\Phi$. The solid line $\Phi(x)=0.73 x^{-0.4}\left\{2\left(1-1.55 x^{-0.4}\right)^{-1.5}-1\right\}$ is calculated using the two-fluid description $\Phi(x)=R_{2 \mathrm{fl}} T$, with $T^{*} \simeq 0.64 H^{0.4}$ and $f_{\mathrm{FL}}(0) \simeq 0.59 H^{0.19}$ at $H=0.45 \mathrm{~T}$. The error bars for $R$ and $R T$ are determined by least squares fits for the nuclear relaxation curves (Fig. 2) using equation (3).

The scaling function $\Phi$ for $\mathrm{YbRh}_{2} \mathrm{Si}_{2}$ contains the two-fluid description $R_{2 \mathrm{fl}}(T)$ as an approximation at each field, which then leads to a good estimate of the $H$-dependence of $T^{*}$ in the lowtemperature limit. Similarly, the scaling relation is considered to be the actual basis for $R_{2 f l}(T)$, but remains hidden in the Ce case owing to the short $\tau$. Moreover, as basic components of the scaling law, the nearly degenerate FL and NFL states are considered to be an essential characteristic of a Kondo lattice at low temperatures, regardless of $\tau$, which is evidently quite long near the QCPT in $\mathrm{YbRh}_{2} \mathrm{Si}_{2}$.

Finally, it should be emphasized that the degenerate FL and NFL states with long $\tau$ for $\mathrm{YbRh}_{2} \mathrm{Si}_{2}$ are not clearly identified at the present time. Even though the two-fluid model is proposed for heavy-fermion formation, such a description may not correspond exactly to the observed FL and NFL states. Perhaps the two coexisting states have a different valence-that is, localized (NFL)
$\mathrm{Yb}^{3+}$ and itinerant (FL) $\mathrm{Yb}^{(3-\delta)+}$ states. For this system, $\tau$ may be long because the $\mathrm{Yb}$ ions are near a valence instability. Thus, the charge degree of freedom may need to be addressed to understand QCPT behaviour in strongly correlated metals - that is, in Kondo lattices as well as for the high- $T_{c}$ cuprates $^{28}$.

\section{Methods}

Single crystals of $\mathrm{YbRh}_{2} \mathrm{Si}_{2}$ were grown by the indium flux method. The starting materials were $\mathrm{Yb}, \mathrm{Rh}$, natural $\mathrm{Si}, 99.3 \%$ enriched ${ }^{29} \mathrm{Si}$ and In. These materials were put in an alumina crucible and sealed in a quartz tube with the stoichiometric composition of $\mathrm{Yb}: \mathrm{Rh}: \mathrm{Si}:{ }^{29} \mathrm{Si}: \mathrm{In}=1: 2: 1: 1: 65$. The ampoule was heated up to $1,200^{\circ} \mathrm{C}$, maintained at this temperature for one day, and cooled to $900^{\circ} \mathrm{C}$ at a rate of $1^{\circ} \mathrm{Ch}^{-1}$, taking about 14 days in total. The excess flux was removed from the crystals by spinning the ampoule in a centrifuge.

High sample purity was confirmed by a small residual resistivity $\rho_{0} \sim 0.99 \mu \Omega \mathrm{cm}$ and a large RRR value $=\rho(300 \mathrm{~K}) / \rho(2 \mathrm{~K})=104$. No magnetic impurity phase was detected in magnetic susceptibility measurements. The 
antiferromagnetic phase transition was observed near $80 \mathrm{mK}$ at zero field. The present result of two coexisting states cannot be interpreted as a second impurity phase because the amount of such a phase is independent of temperature and field. In addition, the small $\Gamma$ and $J_{\text {ex }}$ values for both states cannot be expected for an impurity phase.

One single-crystal piece $\left(6 \times 3 \times 0.1 \mathrm{~mm}^{3}\right)$ was located in the mixing chamber of a dilution refrigerator with an NMR pickup coil for good thermalization of the sample. The standard spin-echo saturation recovery method was used for the determination of spin-lattice relaxation times $T_{1}$. As the resonance linewidth is quite narrow (for example, $\sim 2 \mathrm{kHz}$ at $1.1 \mathrm{~T}$; Supplementary Information), all nuclear spins in the spectrum were uniformly excited by radiofrequency pulses in the $T_{1}$ measurements. The pulse repetition time $t_{\text {rep }}$ in $T_{1}$ measurements was taken sufficiently longer than $T_{1 \mathrm{~L}}$

Received 21 March 2014; accepted 15 August 2014; published online 21 September 2014

\section{References}

1. Gegenwart, P. et al. Magnetic-field induced quantum critical point in $\mathrm{YbRh}_{2} \mathrm{Si}_{2}$. Phys. Rev. Lett. 89, 056402 (2002).

2. Paschen, S. et al. Hall-effect evolution across a heavy-fermion quantum critical point. Nature 432, 881-885 (2004).

3. Friedemann, S. et al. Fermi-surface collapse and dynamical scaling near a quantum-critical point. Proc. Natl Acad. Sci. USA 107, 14547-14551 (2010).

4. Gegenwart, P., Si, Q. \& Steglich, F. Quantum criticality in heavy-fermion metals. Nature Phys. 4, 186-197 (2008).

5. Millis, A. J. Effect of a nonzero temperature on quantum critical points in itinerant fermion systems. Phys. Rev. B 48, 7183-7196 (1993).

6. Si, Q., Rabello, S., Ingersent, K. \& Smith, J. L. Locally critical quantum phase transitions in strongly correlated metals. Nature 413, 804-808 (2001).

7. Coleman, P., Pépin, C., Si, Q. \& Ramazashvili, R. How do Fermi liquids get heavy and die? J. Phys. Condens. Matter 13, R723-R738 (2001)

8. Pépin, C. Kondo breakdown as a selective Mott transition in the Anderson lattice. Phys. Rev. Lett. 98, 206401 (2007).

9. Trovarelli, O. et al. $\mathrm{YbRh}_{2} \mathrm{Si}_{2}$ : Pronounced non-Fermi-liquid effects above a low-lying magnetic phase transition. Phys. Rev. Lett. 85, 626-629 (2000).

10. Custers, J. et al. The break-up of heavy electrons at a quantum critical point. Nature 424, 524-527 (2003).

11. Moriya, T. Nuclear magnetic relaxation in antiferromagnetics. Prog. Theor. Phys. 16, 23-44 (1956).

12. Ishigaki, A. \& Moriya, T. On the theory of spin fluctuations around the magnetic instabilities effects of zero-point fluctuations. J. Phys. Soc. Jpn 67, 3924-3935 (1998).

13. Johnston, D. C. Stretched exponential relaxation arising from a continuous sum of exponential decays. Phys. Rev. B 74, 184430 (2006).

14. Rourke, P. M. C. et al. Magnetic-field dependence of the $\mathrm{YbRh}_{2} \mathrm{Si}_{2}$ Fermi surface. Phys. Rev. Lett. 101, 237205 (2008).

15. Moriya, T. Nuclear magnetic relaxation in antiferromagnetics II. Prog. Theor. Phys. 16, 641-657 (1956).
16. Mila, F. \& Rice, T. M. Analysis of magnetic resonance experiments in $\mathrm{YBa}_{2} \mathrm{Cu}_{3} \mathrm{O}_{7}$. Physica C 157, 561-570 (1989).

17. Stock, C. et al. From incommensurate correlations to mesoscopic spin resonance in $\mathrm{YbRh}_{2} \mathrm{Si}_{2}$. Phys. Rev. Lett. 109, 127201 (2012).

18. Ishida, K. et al. $\mathrm{YbRh}_{2} \mathrm{Si}_{2}$ : Spin fluctuations in the vicinity of a quantum critical point at low magnetic field. Phys. Rev. Lett. 89, 107202 (2002).

19. Kuramoto, Y. \& Kitaoka, Y. Dynamics of heavy electrons (Clarendon Press, 2000)

20. Sichelschmidt, J., Ivanshin, V. A., Ferstl, J., Geibel, C. \& Steglich, F. Low temperature electron spin resonance of the Kondo ion in a heavy fermion metal: $\mathrm{YbRh}_{2} \mathrm{Si}_{2}$. Phys. Rev. Lett. 91, 156401 (2003).

21. Knebel, G. et al. Localization of $4 \mathrm{f}$ state in $\mathrm{YbRh}_{2} \mathrm{Si}_{2}$ under magnetic field and high pressure: Comparison with $\mathrm{CeRh}_{2} \mathrm{Si}_{2}$. J. Phys. Soc. Jpn 75, 114709 (2006)

22. Watanabe, S. \& Miyake, K. Quantum valence criticality as an origin of unconventional critical phenomena. Phys. Rev. Lett. 105, 186403 (2010).

23. Abrahams, E., Schmalian, J. \& Wölfle, P. Strong-coupling theory of heavy-fermion criticality. Phys. Rev. B 90, 045105 (2014).

24. Nakatsuji, S. et al. Two fluid description of the Kondo lattice. Phys. Rev. Lett. 92, 016401 (2008).

25. Yang, Y-F. \& Pines, D. Universal behaviour in heavy-electron materials. Phys. Rev. Lett. 100, 096404 (2008)

26. Sakai, H. et al. Magnetic-field-induced enhancements of nuclear spin-lattice relaxation rates in the heavy-fermion superconductor $\mathrm{CeCoIn}_{5}$ using ${ }^{59} \mathrm{Co}$ nuclear magnetic resonance. Phys. Rev. Lett. 107, 137001 (2011).

27. Joss, W. et al. Observation of the magnetic field dependence of the cyclotron mass in the Kondo lattice $\mathrm{CeB}_{6}$. Phys. Rev. Lett. 59, 1609-1613 (1987).

28. Wu, T. et al. Magnetic-field-induced charge-stripe order in the high-temperature superconductor $\mathrm{YBa}_{2} \mathrm{Cu}_{3} \mathrm{O}_{y}$. Nature 477, 191-194 (2011).

\section{Acknowledgements}

We are grateful for stimulating discussions with S. Watanabe, K. Izawa, K. Ishida, H. Ikeda, J. P. Brison, Y-F. Yang, Z. Fisk and K. Kubo. A part of this work was supported by Grants-in-Aid for Scientific Research on Innovative Areas Heavy Electrons (No. 20102006 and No. 20102007) from MEXT, and the REIMEI Research Program of JAEA.

\section{Author contributions}

S.K. designed and carried out NMR measurements and analysis of results, and prepared the paper. H.S. and Y.T. participated in the measurements and analysis. G.L., T.D.M. and G.K. developed the single-crystal sample and performed sample characterization. J.F. and R.E.W. participated in the analysis.

\section{Additional information}

Supplementary information is available in the online version of the paper. Reprints and permissions information is available online at www.nature.com/reprints. Correspondence and requests for materials should be addressed to S.K.

\section{Competing financial interests}

The authors declare no competing financial interests. 